as to render it practically unreliable. I agree with Richards that, so far as it goes, it is a good local application, and as such it ought to be used; or, in its absence, tannic acid or liquor potasso might be resorted to with the same object; but as a constitutional remedy, as a physiological antidote, it is powerless, like all others that have been tried and failed to do good; and Lacerda himself, athough he attributes the highest value to it as a chemical antidote, both as a yowerful oxdisin $\mathrm{g}$ agent and by the action of the potash, says : "As to the idea of finding a physiological antidote for snakeroisoning, I entirely agree with you that it is a utopia." 3

After careful consideration, fully admitting that in permanganate of potash we have an agent which can chemically neutralise snake-poison, as indeed was shown by Dr. Brunton and myself in 1875 , I do not ascertain that more has been done than to draw attention to a local remedy already wellknown as a chemical antidote, the value of which depends on its efficient application to the contaminated part (which Dr. Wall has pointed out is too uncertain to be reliable), and we are still as far off an antidote as ever, whilst the remarks made by me in 1868 are as applicable now as they were then. They were as follows:- "To conceive ci an antidote (as that term is usually understood) we raust imagine a substance so subtle as to follow, overtake, and neutralise the venom in the blood, and that shall have the power of counteracting or neutralising the poisonous and deadly influence it has exerted on vital force. Such a substance has still to be found, nor does our present experience of drugs give hopeful anticipation that we shall find it; but I repeat that where the poisonous effects are produced in a minor degree, or where the secondary consequences are to be dealt with, we may do much to aid the narural powers in bringing about recovery."

In conclusion, fully acknowledging the value of recent reisuarches, I would express a hope that the subject may receive further vigorous investigation, and that efforts may be prosecuted especially in the direction of search for some method of increasing elimination of the poison, of ascertaining the exact nature of the lesions of the nervous system and blood, and how far they are removable. As to local measures, with the view of preventing entry of the virus into the circulation, and of neutralising it in situ, improvements on the present method may be sought for.

As to advance in the investigation of the physiolngical and chemical aspects of the question, much may still be done, as also in respect of the chemistry and mieroscopical characters of the virus itself, and the blnod and tissues of the poisoned. But these inquiries, of such importance to the human race, can, I fear, make but little further progress whilst the present restriction on physiological research continues to be maintained.

A review of the subject of snake-poisoning would be quite incomplete without acknowledgment of the valuable labours of such Indian observers as Drs. Shortt, Nicholson, Ewart, V. Richards, Wall, Stradling, and Stewart, who have added mateiially to our knowledge. Also of Dr. Halford in Australia, Dr. de Lacerda in Kio, Dr. T. L. Brunton in London, and of Drs. Weir Mitchell and Reichart in America, who are even now engaged in a most important and muchneeded investigation unto the chemistry of the poison and the condition of the blood and tissues of the poisoned.

\section{IMPROVEMENTS IN SYRINGES.}

\section{BY R, FITZROY BENHAM, M.R.C.S., \&C.}

HAVING observed for some time past the great inconvenience and expense one experiences in having to use various forms of syringes for the innumerable purposes for which they are required, both in medical and surgical cases, I have been induced, in consequence, to modify the mechanism of the extremity of the ordinary type of syringe, so that one syringe may be used for numberless different purposesfor instance, whether it be required to wash out an abscess cavity or to syringe a wound, to aspirate the bladder or to perform paracentesis, for the transfusion of fluids, or the injection of an enema, \&c. The modification to which I refer

3 Dr. Lacerda has probably operated only with crotaline snakes ; and if so, bas not had the opportunity of observing the difference of action exerted by colubrine poison. In my experience repeated experiments show that direct general contamination may follow even when no large vein has been penetrated. will, I have no doubt, be readily understood from the diagrams. A is a hollow T. shaped piece of metal, which is adapted to the ordinary syringe in the pla:e of its nozzle, c. The handle, B, is attached to a coned plug in which there is a rectangular aperture, so that by turning the handle $B$, either to the left or to the right, the way, so to speak, is opened to eitber the inlet or the outlet aperture, or vice versa, as de. sired. To the end of each transverse extremity of $A$ a piece of thick rubber tubing is attached, as in the ordinary aspirating or flute-action syringes. To one extremity of the tubing a hollow weight is connected, whilst at the other varions attachments may be adjusted. For instance, if the syringe be required for the purpose of washing out a cavity or syringing a wound, the nozzle, $\mathrm{C}$, of the syringe may be connected with the extremity of the flexible tube, and thrs fluid may be driven in either direction by simply turning the bandle, $\mathbf{B}$, to the left or to the right, at will. Again, if it be required to perform paracentesis, or to aspirate the bladder the nozzle, $C$, may be replaced by an ordinary cannula and trocar, D, as shown in the diagram. Thus it may be seen that by simply applying various attachments to the extremities of the tubing any ordinary syringe may be at onee adapted for the various purposes. Apart from the advantages to be obtained as above described, by simply applying this modification or addition $A$ to an ordinary syringe, it may be mentioned : 1st. That the direction of the inlet or the outlet being in a line, instead of rectangular, as in the case of the

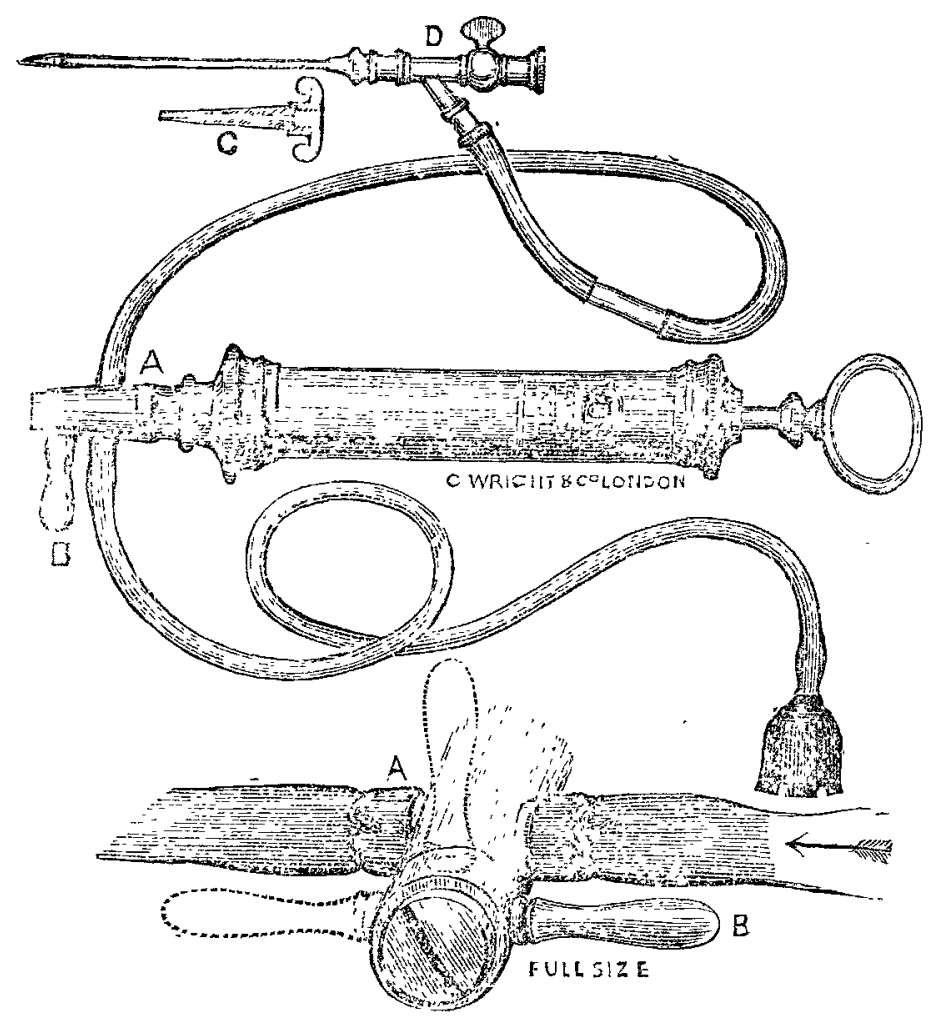

ordinary stomach pump with its flute action mechanism, it is impossible for one aperture to be opened before the other is completely closed, notwithstanding they may be of considerable size, 2nd. No matter how small the extremity of the nozzle, c, may be, it does not impede the flow of the fluid when the syringe is being refilled-a point, I venture to think, of considerable moment where a large quantity of fluid is required, as in the case of syringing a wound after operation, thus saving time and the annoyance of unscrewing or detaching the nozzle from the extremity of the syringe before refilling the latter. 3rd. When the nozzle of the syringe is at once in situ it may be allowed to remain 80 until it is no longer required, whereas with the ordinary syringe it must be replaced every time it is refilled. 4th. As there are no valves or complicated mechanism the working parts cannot possibly get out of order or become corroded 5th. The attachments, A, may be applied to all existing syringes by simply causing the size of its screw to be made to correspond with the nozzle, while other portions, such as a cannula and trocar, may be adapted at will. In conclusion I may state, for the information of the profession, that Mcesrs. C. Wright and Co., of 108, New Bond-street, London, have in stock various sizes of the attachments as above described and that on their receiving an ordinary syringe they will adapt them accordingly.

Baron's Court, S.W. 